war, weil die alte politische Klasse sich außerstande zeigte, die genuinen Probleme der Weimarer Republik zu lösen. Anders gesagt: Auch in Deutschland ist der Nationalsozialismus "nicht vom Himmel gefallen«, wie bereits vor ihm Silone in seiner Untersuchung über die historische Genese des italienischen Faschismus herausfand, sondern aufgrund der komplexen politischen und gesellschaftlichen Krise der Weimarer Republik.

Für Borkenau war der Totalitarismus bereits qua definitionem bzw. Selbstverständnis der natürliche Gegner, genauer: der existenzielle Feind der liberalen oder pluralistischen Demokratie, mit dem eine friedliche Koexistenz ausgeschlossen war. Einerseits weil die Feindsuche zur Obsession und zum circulus vitiosus des Totalitarismus wurde und mithin ein friedliches Zusammenleben mit anderen, zumal demokratischen Staaten nicht möglich war. Andererseits war der Totalitarismus dem ideologischen Selbstverständnis nach die Antithese zu den Demokratien, da er dem Staat, der Partei und der totalitären Massenbewegung den absoluten Vorrang einräumte und er sich das "Ende des Subjektes « und die radikale Beseitigung aller persönlichen sowie staatsfreien Freiheitsrechte zum Ziel gesetzt hatte.

Neben der konkreten Beschreibung und Analyse des stalinistischen Herrschaftsregimes lag die Bedeutung der Renegaten für die »klassischen« Totalitarismustheorien zudem vor allem darin, dass sie den Fokus einerseits auf die für die einzelnen Studien zentralen Kategorien wie »Einparteienregime«, »Geheimpolizei«, »totalitäre Ideologie«, »(permanenter) Terror«, »Konzentrationslager«, »Feindobsession«, »permanente Revolution« etc. richteten. Andererseits haben sie die differentia specifica zwischen dem italienischen Faschismus und dem Nationalsozialismus sowie dem Sowjetkommunismus hervorgehoben. Schon Borkenau subsumierte in The Totalitarian Enemy ausdrücklich nur den Nationalsozialismus und den Sowjetkommunismus unter das Totalitarismusmodell. Zudem arbeiteten die Renegaten in den autobiografischen Erfahrungsberichten ex negativo die qualitativen Unterschiede heraus, die zwischen einem totalitären und einem faschistischen Herrschaftsregime bestanden - bei allen zweifelsohne vorhandenen Gemeinsamkeiten -, dass nämlich der Totalitarismus eine Verbrechensdimension entwickelte, die dem italienischen Faschismus unter Mussolini gewissermaßen fremd war. Insbesondere der amerikanische Ex-Trotzkist Macdonald unterstrich bereits 1944 explizit und implizit den einzigartigen Herrschaftscharakter des nationalsozialistischen Totalitarismus in Bezug auf die Ermordung der europäischen Juden. Vergleichbare Verbrechen hatte der italienische Faschismus nicht begangen. Vor diesem Hintergrund gingen Arendt und Friedrich später in ihren »klassischen« totalitarismustheoretischen Studien von den qualitativen Differenzen zwischen dem italienischen Faschismus auf der einen Seite und dem nationalsozialistischen sowie sowjetkommunistischen Totalitarismus aus.

\title{
3.4 Die Rolle der Renegaten im Monat
}

Vor dem Hintergrund ihrer frühzeitigen theoretischen und politischen Auseinandersetzung mit den totalitären Herrschaftssystemen des Nationalsozialismus und des Sowjetkommunismus kann auch im Folgenden die spezielle Rolle der zahlreichen ehemaligen kommunistischen (Partei-)Intellektuellen in der Zeitschrift Der Monat verdeutlicht werden. Aufgrund der zum Teil frühzeitig einsetzenden Ausein- 
andersetzung mit dem Nationalsozialismus und dem Sowjetkommunismus waren die Renegaten sozusagen prädestiniert, Vergleiche zwischen den beiden totalitären Herrschaftssystemen zu ziehen und Gemeinsamkeiten bzw. Unterschiede hervorzuheben. ${ }^{215}$ Nachdem der Nationalsozialismus von den Alliierten erst unter der Mobilisierung aller staatlichen Ressourcen militärisch besiegt werden konnte, sollten die vielen Renegaten in der Hochphase des Kalten Krieges im Monat allerdings in erster Linie die vielschichtige Auseinandersetzung mit dem Kommunismus fortführen bzw. den Kampf mit dem Stalinismus wiederaufnehmen. ${ }^{216}$ Dieser zum Teil existenzielle Dimensionen annehmende Kampf gegen die Sowjetunion und die osteuropäischen Satellitenstaaten wurde intellektuell, politisch, kulturell und literarisch geführt. Für die Renegaten wiederum stand fest, dass in Anbetracht des in Trümmern liegenden >Dritten Reiches die zentrale persönliche politische Aufgabe darin bestand, den aktuellen Totalitarismus zu bekämpfen. Nicht zuletzt die antikommunistischen Renegaten erkannten frühzeitig die zeitgenössische Gefahr, die, vor dem Hintergrund der Anziehungskraft des Kommunismus insbesondere in Europa, von der Sowjetunion nach dem Ende des Zweiten Weltkrieges ausging. ${ }^{217}$ Angesichts der persönlichen Erfahrung mit der kommunistischen Weltanschauung und der Kommunistischen Partei waren sich in allererster Linie die Renegaten aufgrund der "Insiderkenntnisse« der facettenreichen Gefahr bewusst, die von Moskau ausging. Einerseits gingen sie von der (totalitarismustheoretischen) Erkenntnis aus, dass für die demokratischen Staaten mit dem sowjetischen Imperium Stalins kein friedliches Zusammenleben möglich war. Andererseits besaßen die Renegaten das historische, theoretische und politische Wissen, um das sowjetkommunistische Herrschaftssystem zu beschreiben und zu analysieren und beispielsweise gegen das in weiten Kreisen der Intellektuellen, der Kirchen und des Bildungsbürgertums in Deutschland vorhandene Bild einer Sowjetunion zu kämpfen, die sich den Antifaschismus und die Friedenspolitik auf ihre politpropagandistische Fahnen geschrieben hatte. Insbesondere hinsichtlich des Wiederauflebens des aus den 1930er-Jahren stammenden Konzeptes der »Volksfrontpolitik« Moskaus und der damit zusammenhängenden »Friedenskongresse« Moskaus in Breslau, New York

215 Siehe exemplarisch den aus totalitarismustheoretischer Perspektive geschriebenen Beitrag Borkenau, Zwischen Rot und Braun.

216 Wie gezeigt, war aufseiten der US-Administration in der Zeit der politischen und militärischen Zusammenarbeit mit der Sowjetunion gegen den Nationalsozialismus, die Auseinandersetzung mit dem Kommunismus im Allgemeinen und dem Stalinregime im Besonderen nur »auf Eis«gelegt. Insbesondere am Ende des Zweiten Weltkrieges bzw. im Verlauf der diplomatischen Zusammenkünfte der Vereinigten Staaten mit der Sowjetunion kam auf institutioneller Seite die facettenreiche Auseinandersetzung mit dem Kommunismus wieder in Gang. Hierzu gehörte auch die Erkenntnis in der US-Administration, ehemalige Kommunisten im Kampf gegen den sowjetischen Totalitarismus zu gewinnen. Aus diesem Grund konnte später angesichts der bipolaren Konfrontation zwischen den westlichen Staaten und der Sowjetunion der FBI-Chef Hoover schreiben: »Der Exkommunist ist heute eine unserer schlagkräftigsten Waffen im Kampf gegen den Kommunismus.«(Zit. n. Roloff, Exkommunisten, S. 16)

217 Beispielsweise schrieb Allemann aus Anlass des Todes des Berliner Bürgermeisters Ernst Reuter am 29. September 1953 in Berlin rückblickend, dass u. a. der Exkommunist Ernst Reuter sehr früh den »Expansionswillen des Kreml« vorausgesehen hatte und »daß an die Stelle des deutschen nun der sowjetische Imperialismus als Weltgefahr und permanente Friedensbedrohung getreten war« (F[ritz] R[ené] Allemann, Der Türmer von Berlin, in: Der Monat 6 (1953), H. 62, S. 116-122, hier S. 121). 
und Paris sowie des 1947 stattgefundenen »Ersten deutschen Schriftstellerkongresses« im geteilten Berlin läuteten - salopp formuliert - bei den »Altmeistern des Agitprop« (Furet) wie Koestler oder Sperber die Alarmglocken. Denn insbesondere nach den Abwürfen der Atombomben auf Hiroshima und Nagasaki schien vielen Schriftstellern, Künstlern und Wissenschaftlern in Deutschland Moskau Ende der 1940er-Jahre als der Hort des Friedens.

Beispielsweise hob Borkenau auf dem Berliner »Kongreß für kulturelle Freiheit« im Juni 1950 - auch vor dem Hintergrund des soeben ausgebrochenen Koreakrieges die zentrale Bedeutung der Renegaten im Kampf mit dem Kommunismus und dem "Stalin-Regime« hervor. Sie resultierte aus der Tatsache, so die mit Pathos vertretene Auffassung Borkenaus, dass

viele Tausende von politisch und literarisch aktiven Menschen durch den Kommunismus hindurchgegangen sind und mit ihm gebrochen haben. Diese Menschen wissenund sie wissen es mit einer Deutlichkeit und Unbeirrbarkeit wie kaum jemand sonst-, daß der Kampf gegen die kommunistische Drohung die alles überschattende Hauptaufgabe der Cegenwart ist. ${ }^{218}$

Auch Berlins regierender Bürgermeister Ernst Reuter begründete den Kampf für die politische und kulturelle Freiheit und gegen die Sowjetunion ausdrücklich mit seinen persönlichen Erfahrungen mit dem Kommunismus. Eingedenk der »Berliner Blockade« durch Moskau sagte der ehemalige Generalsekretär der KPD in seiner Begrüßungsrede auf der Eröffnungskundgebung des Berliner Kongresses am 26. Juni 1950 in Anbetracht der bestehenden aktuellen Gefahr:

Viele unter uns haben die große Tragödie unserer Zeit darum so tief durchlebt und so lebendig erfaßt, weil sie im Suchen nach der Wahrheit, im Suchen nach dem Recht selber all den Versuchungen unterlegen gewesen sind, die aus der revolutionären Entwicklung des Maschinenzeitalters, des Massenmenschen und des kollektiven Zusammenlebens unvermeidlich über uns kommen mußten. Vielleicht konnten überhaupt nur Menschen, die der Versuchung unterlegen gewesen sind, dem Irrlicht aus dem Osten zu folgen, mit letzter plastischer, jeden überzeugender Deutlichkeit die Größe der

218 Franz Borkenau, Rückkehr zu den alten Werten?, in: Der Monat 2 (1950), H. 22/23, S. 417. In einer Diskussionsrunde replizierte Borkenau auf den von Adolf Grimme vorgebrachten, in Richtung Koestler zielenden Vorwurf, dass dieser »sich zwischen der sScylla des politischen Konvertitentums und der Charbydis der Dialektik durchgewunden« habe (ebd., S. 467 f.). Borkenau vertrat dagegen die Auffassung: »daß gerade die Konvertiten, die den Kommunismus aktiv durchlebt haben, heute in der Demokratie einen ganz besonderen Beitrag zu geben haben. Und weiter: »lch selbst bin Konvertit vom Kommunismus und ich bin stolz darauf. Denn gerade wir wissen, daß die kommunistische Gefahr eine totale Gefahr ist, vor deren Unerbittlichkeit es kein Ausweichen gibt. Wie viele von uns denken heute: Frieden um jeden Preis. Aber es gibt keine Beschwörungsformel. Das Regime wird zusammenbrechen, oder sich als zerstörende Macht auf die Welt stürzen.«(Ebd., S. 468). Siehe auch Borkenau im Nachwort zu: Ein Gott der keiner war. Arthur Koestler, Ignazio Silone, Richard Wright, André Gide, Louis Fischer, Stephen Spender schildern ihren Weg zum Kommunismus und ihre Abkehr, S. 297. Vgl. Sidney Hook, Out of Step. An unquit Life in the 20th Century, New York 1987, S. 438. 
Gefahr aufzeigen, in die die Welt zu geraten droht, eine Gefahr, die heute weniger denn je gebannt ist. ${ }^{219}$

Eingedenk der historischen Erkenntnis, die unter anderem auch von Borkenau in seiner Totalitarismusstudie hervorgehoben wurde, dass die Ausbreitung des Totalitarismusphänomens nur möglich war, weil die westlichen Gesellschaften den »geschichtlichen Boden « bereitet hatten, insistierten neben anderen Autoren des Monat vor allem auch die Renegaten in ihren Zeitschriftenbeiträgen auf die Tatsache, dass der Erfolg des sowjetischen Kommunismus nicht zuletzt von den westlichen Staaten selber abhängt. Das heißt, auch der Innen- und Außenpolitik der westlichen demokratischen Staaten wurde in diesem Zusammenhang eine essenzielle Bedeutung zuerkannt. Zum einen gingen einige ehemalige Kommunisten von der Prämisse aus, dass auch die westlichen Gesellschaften nicht vor der totalitären Gefahr gefeit wären und folglich die demokratischen Freiheitsrechte uneingeschränkt verteidigt werden müssen, sodass man für einen sozialen und politischen Reformprozess in den westlichen Demokratien plädierte. ${ }^{220}$ Zum anderen rekurrierten - vor dem Hintergrund der westlichen Appeasementpolitik gegenüber dem Nationalsozialismus und des vertraglichen Nichtangriffspaktes zwischen den beiden weltanschaulichen »Todfeinden« Deutschland und Sowjetunion - unter anderem einige Renegaten auf einer genuin antitotalitären westlichen Diplomatie, die sich der kommunistischen Expansionspolitik gewahr ist. $^{221}$

Nach ihrem Ablösungsprozess von der kommunistischen Bewegung und der Trennung von der Kommunistischen Partei, der bei den allermeisten Renegaten mit dem existenziellen Verlust der "politischen Heimat" (Rohrwasser) korrespondierte, entwickelte sich die Zeitschrift Der Monat bei ebendieser Personengruppe auch zu einer »Zweiten Heimat«. Im Monat konnten persönliche, politische und theoretische Probleme zur Diskussion gestellt werden, die nicht zuletzt für die politische Linke und für die (Links-)Intellektuellen von hoher Bedeutung war. Beispielsweise konnten die Renegaten im Monat die Diskussion weiterführen, die einige Jahre zuvor Orwell, Borkenau und Burnham anlässlich ihrer jeweiligen Buchveröffentlichungen schon in Gang gesetzt hatten. Die drei Intellektuellen hatten sich nicht nur qua Kritik und Selbstkritik das politische Scheitern der bolschewistischen Revolution von 1917 ein-

219 Ernst Reuter, in: Der Monat 2 (1950), H. 22/23 S. 342.

220 Durch ihre vornehmlich auf die Sowjetunion ausgerichtete Beschreibung und Analyse des Totalitarismus entfalteten die Renegaten im Monat auch ex negativo eine Demokratietheorie, denn die totalitäre Gesellschaft wurde zu einem gesellschaftlichen »Schreckbild«apostrophiert. Fritz René Allemann versuchte beispielsweise zu verdeutlichen, wie aus dem ehemaligen linken Sozialrevolutionär Sol Levitas ein Demokrat und überzeugter Kämpfer für die »Freiheit«werden konnte. Levitas, der bis zu seinem Tod Herausgeber der einflussreichen antistalinistischen amerikanischen Wochenzeitschrift The New Leader war, war Allemann zufolge früher russischer Revolutionär und Menschewik und mehrere Jahre in »bolschewistischen Gefängnissen« inhaftiert und für ihn seitdem gewissermaßen bekehrt. Gleichwohl hatte seiner Auffassung nach die von Levitas in der amerikanischen Öffentlichkeit artikulierte Gegnerschaft gegenüber der Sowjetunion »nichts von der Verbissenschaft des professionellen Antikommunisten an sich, und McCarthy war ihm genauso ein Greuel wie alle Leute auf der anderen Seite, die im Namen der >Freiheit، der Freiheit den Garaus zu machen suchten.«(Sol Levitas †, in: Der Monat 13 (1961), H. 149, S. 90 f.)

221 Siehe exemplarisch Löwenthal, Die Freiheit und der Konflikt der Westmächte. 
gestanden, sondern auch die theoretische Feststellung machen müssen, dass der Zusammenbruch des Kapitalismus nicht - wie es die marxistische Theorie prognostizierte - automatisch zum Sozialismus führte. Anders gesagt: Der Monat war für (ehemalige) Repräsentanten der politischen Linken ein (Veröffentlichungs-)Medium der Selbstverständigung über den genuinen Zusammenhang zwischen marxistischer Theorie, sozialistischem Ökonomiemodell, Russischer Revolution, sowjetkommunistischer Einparteienherrschaft sowie totalitärem System. ${ }^{222}$

Darüber hinaus thematisierten die Renegaten im Monat den Desillussionierungsprozess und die individuellen Wandlungen seit Mitte der 1930er-Jahre, sodass es ihnen möglich wurde, mit dem Kommunismus, der Kommunistischen Partei sowie einem idealisierten Bild der Sowjetunion zu brechen. Stellvertretend für zahlreiche Intellektuelle beschrieben zum Beispiel Koestler oder Silone, warum sie mehrere Jahre lang auf der Grundlage der kommunistischen Weltanschauung bzw. des kommunistischen »Glaubens« von der Überlegenheit des sowjetischen Gesellschaftsmodells überzeugt waren. Mit Blick auf die aktuellen Parteiideologen und die »fellow traveller«, die auch noch nach dem Zweiten Weltkrieg das »russische Wunder« und die »Überlegenheit des sozialistischen Weltsystems " priesen, unternahmen einige der international bekanntesten ehemaligen kommunistischen Intellektuellen im Monat auch den Versuch zu erklären, warum sie - unter dem Einfluss der marxistischen »Heilslehre« stehend und auf Grundlage eines manichäischen antifaschistischen Weltbildes - durch einen lange Zeit nicht zu erschütternden Glauben immer wieder den »Mythos Sowjetunion« heraufbeschwörten und in Moskau - infolge der bolschewistischen Revolution von 1917 - das historisch legitimierte genuine Zentrum des internationalen Proletariats sahen. Warum sie trotz zum Teil persönlicher Reisen in das "gelobte Land«, trotz ökonomischen Desasters des "Großen Planes«, trotz Konzentrationslagern, Schauprozessen und Terror nachgerade »vorsätzlich blind« waren und nichtsdestotrotz manchmal über einen Zeitraum von mehreren Jahren von dem »Gott der keiner war« überzeugt und fasziniert waren. ${ }^{223}$

\section{Porträts einiger Exponenten des Monat Vorbemerkung}

In den beiden vorangegangenen Kapiteln sollte auch gezeigt werden, was die totalitäre Erfahrung historisch für die betroffenen Individuen bedeutete. Dass nämlich die lebensgeschichtliche Erfahrung der Verfolgung durch den deutschen Nationalsozialismus und den sowjetischen Kommunismus nicht nur mit Flucht, sondern in

222 Siehe hierzu exemplarisch: James Burnham, Wesen des Kommunismus, in: Der Monat 1 (1948), H. 2 , S. 18-29; Paul Kecskemeti, Jenseits des Sozialismus, in: Der Monat 1 (1948), H. 3, S. 103-106 (Rezension), sowie den Leserbrief hierzu: Paul Sering, Planung und Freiheit, in: Der Monat 1 (1949), H. 5 , S. 106-109; Bertram D. Wolfe, Trotzki über Stalin, in: Der Monat 1 (1948), H. 3, S. 106-111 (Rezension); die Veröffentlichung von George Orwells Kurzroman Farm der Tiere im Monat (1 [1949], H. 5, S. 38-54, H. 6, S. 10-24, sowie H. 7, S. 34-43); Franz Borkenau, Von Marx bis Stalin, in: Der Monat 1 (1949), H. 5 , S. 98-100 (Rezension); Arthur Koestler, Demi-vierges und gefallene Engel. Der gefährliche Flirt mit dem Totalitarismus, in: Der Monat 1 (1949), H. 11, S. 119-121 (Rezension); vgl. auch Sidney Hook, Die Zukunft der demokratischen Linken, in: Der Monat 1 (1949), H. 5, S. $13-17$.

223 Siehe bes. die Reihe»Der Gott, der keiner war«bzw. »Ein Gott hat versagt«. 